\title{
Case Report and Review of the Literature
}

\section{Mature Teratoma in the Third Ventricle: A Case Report and Review of the Literature}

\author{
Yanire Sánchez-Medina*, Eric Robles Hidalgo, Jaime Domínguez, Baez and Luis Gómez Perals
}

Department of Neurosurgery, Nuestra Señora de Candelaria University Hospital, Tenerife, Spain

\begin{tabular}{l} 
A R T I C L E I N F O \\
\hline Article history: \\
Received: 9 January, 2021 \\
Accepted: 25 January, 2021 \\
Published: 4 February, 2021 \\
\hline Keywords: \\
Teratoma \\
third ventricle \\
ventricular tumor \\
intracranial \\
germ cell tumors
\end{tabular}

\begin{abstract}
A B S T R A C T
Introduction: Germ Cell Tumors (GCT) represent less than $4 \%$ of primary brain tumors. They comprise Germaniums, Non-Germinomatous Germ Cell Tumors and Teratomas. Teratomas represent less than $20 \%$ of intracranial GCT. They are tumors of multipotential cells derived from all 3 germ cell layers, frequently arising in midline structures, most commonly in the pineal and suprasellar regions, with a clear excess of male cases and frequently found in children and young adults. We report a case of a mature teratoma in the third ventricle in a 37-year-old male.

Case Report: We report a case of a 37-year-old male with a history of headache lasting up to 9 days and refractory to pharmacological treatment. The CT scan revealed a $20 \mathrm{~mm}$ round hypodense lesion in the anterior third ventricle, with a punctate hyperdensity in the inferior pole causing biventricular hydrocephalus with no periventricular lucency and the MRI showed a well-defined encapsulated mass lesion attached to the roof of the third ventricle, isointense in T1WI with circumferential enhancement and hyperintense in T2WI. Gross total resection was performed. Histopathologic evaluation revealed a mature teratoma. There was no evidence of recurrence on follow up MRI at 2 years.

Conclusion: Intracranial teratomas typically originate in midline structures from optic chiasm to pineal region. Presentation after the first two decades of life is exceptional. Complete surgical resection is the only curative treatment for pure mature teratomas. We report the case of a mature teratoma in a 37 -year-old male with unusual radiological findings.
\end{abstract}

() 2021 Yanire Sánchez Medina. Hosting by Science Repository.

\section{Introduction}

Intracranial teratomas are rare neoplasms accounting for $0.5-1 \%$ of all intracranial tumors, with a higher incidence in children representing 2 $4 \%$ of pediatric primary brain tumors [1-3]. They often arise in midline structures, commonly in the pineal and suprasellar region but has rarely found in the third ventricle. Presentation after the first two decades of life is exceptional. We describe a case of a mature teratoma in the third ventricle of a 37 -year-old male patient.

\section{Case Report}

A 37-year-old male without any relevant medical history, presented to the hospital with 9 days of bifrontal headache, unresponsive to medical treatment and prone to wake him up late at night. Physical finding was bilateral blurring of nasal border of the optic disc. An unenhanced CT scan revealed a $20 \mathrm{~mm}$ round hypodense lesion in the anterior third ventricle, with a punctate hyperdensity in the inferior pole, causing obstruction of both foramen of Monro with biventricular hydrocephalus without periventricular lucency (Figure 1). MRI with gadolinium showed a well-defined encapsulated mass lesion attached to the roof of the third ventricle, isointense in T1WI with circumferential enhancement and hyperintense in T2WI (Figure 2).

Two days after his admission he was taken to surgery to have a right frontal craniotomy and a transcortical transventricular transforaminal approach leading to gross total resection of a globule shaped lesion attached to the septum pellucidum and right fornix and filled with

${ }^{*}$ Correspondence to: Yanire Sánchez Medina, Department of Neurosurgery, Nuestra Señora de Candelaria University Hospital, Carretera del Rosario s/n, 38010, Santa Cruz de Tenerife, Spain; E-mail: yanire.sm@hotmail.com

(C) 2021 Yanire Sánchez Medina. This is an open-access article distributed under the terms of the Creative Commons Attribution License, which permits unrestricted use, distribution, and reproduction in any medium, provided the original author and source are credited. Hosting by Science Repository.

http://dx.doi.org/10.31487/j.SCR.2021.02.04 
creamy yellow contents. An External ventricular drainage was placed during the procedure that was kept closed and withdrawn 24 hours after the surgery. There was complete resolution of patient's headache without neither memory impairment nor any other neurological deficit. Patient had two separate CT scans showing a complete resection of the lesion and resolution of the hydrocephalus (Figure 3). Histopathologic examination revealed a mature teratoma formed by a cystic cavity lined by respiratory epithelium with mature bone in its interior (Figure 4). No further treatment was prescribed, and the patient was discharged one week after the craniotomy. There was no evidence of recurrence after 4 years on follow up MRI and the patient was asymptomatic.

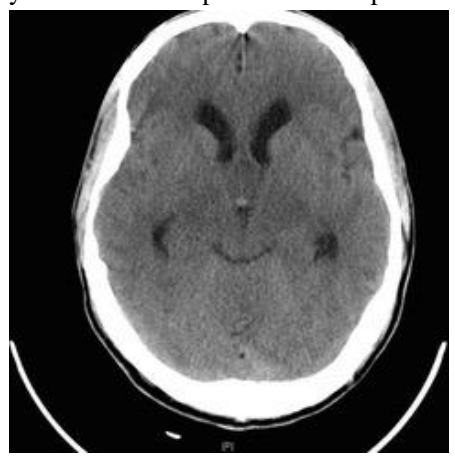

Figure 1: Preoperative CT scan revealed a $20 \mathrm{~mm}$ round hypodense lesion in the anterior third ventricle.

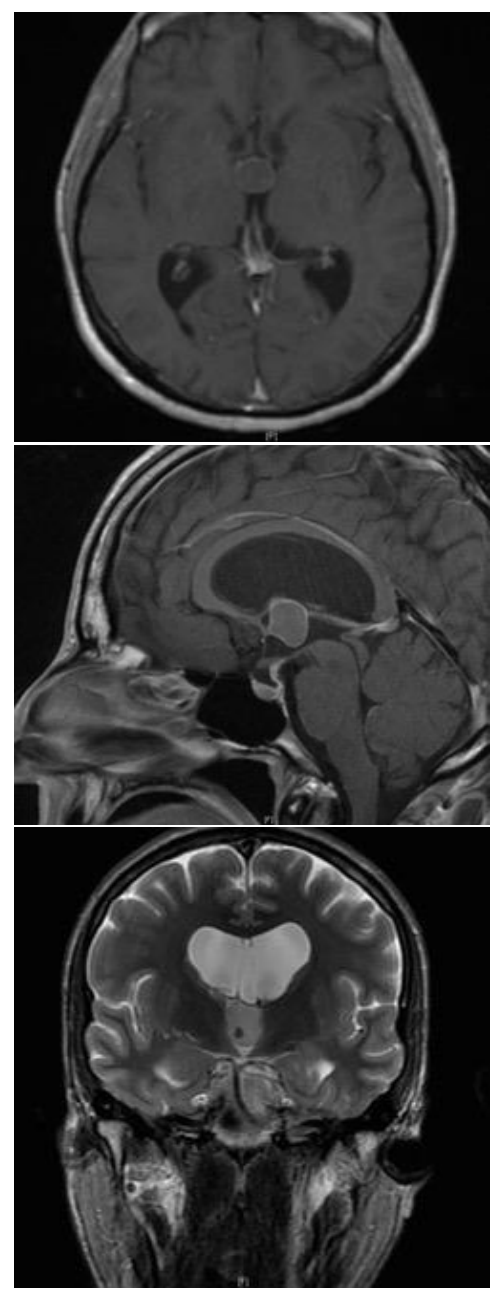

Figure 2: MRI with gadolinium showed a well-defined encapsulated mass lesion attached to the roof of the third ventricle.
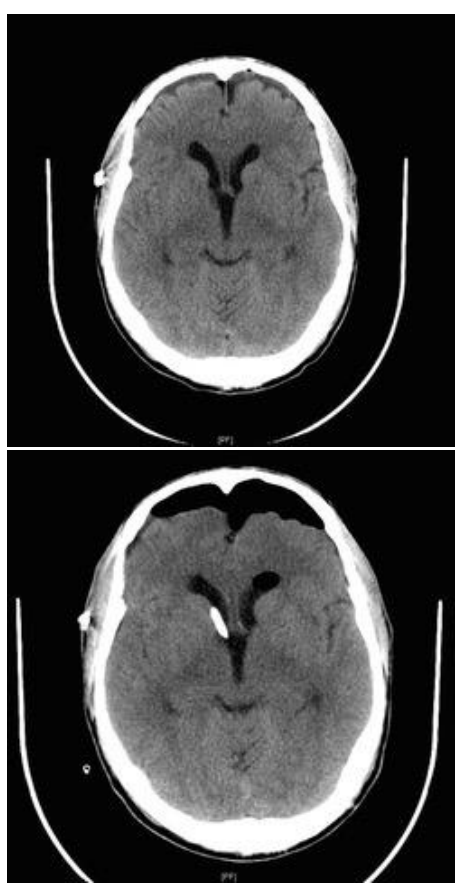

Figure 3: Postoperative CT scan showing complete remove of previous tumor and a close EVD.

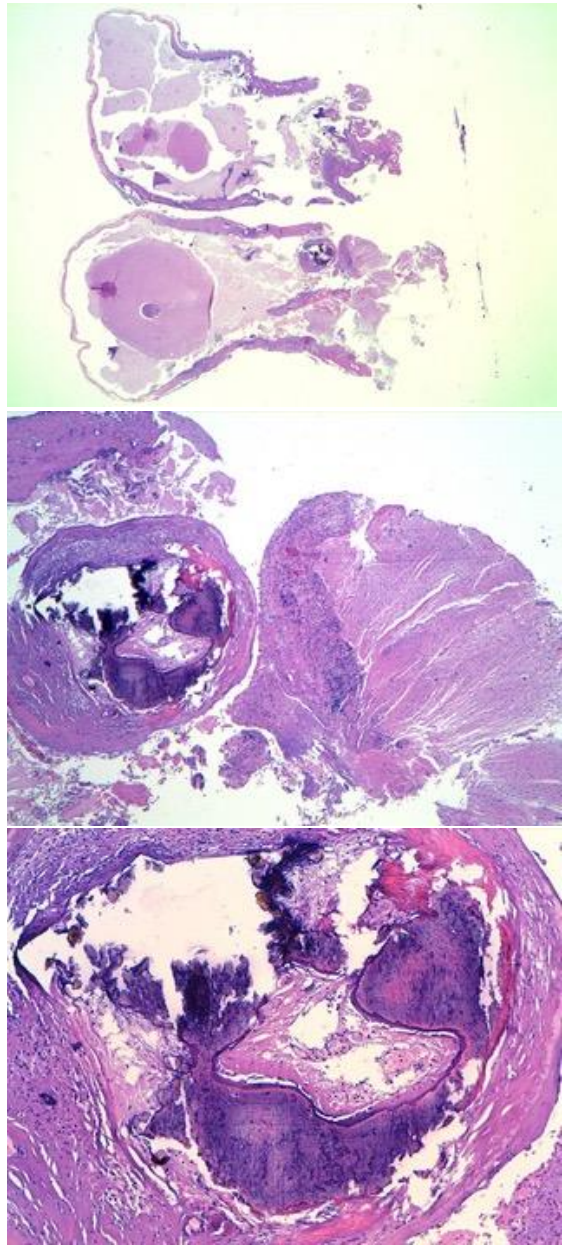

Figure 4: Histopathologic examination showing a cystic cavity lined by respiratory epithelium with mature bone in its interior (4.2 mature bone $\mathrm{x} 10)$. 


\section{Discussion}

Intracranial Teratomas are a subset of Central Nervous System Germ Cell Tumors (GGT) representing extragonadal homologues to gonadal germinal neoplasm. GCT accounts for approximately $3 \%$ of all primary intracranial tumors, with a higher frequency in pediatric series, reaching $15 \%$ of pediatric brain tumors in Asian countries [1, 2, 4, 5]. They typically originate in midline structures, with a median age at diagnosis of 10-14 years old [4]. Males are more often affected, especially for tumors in the pineal region [4]. Clinical symptoms depend on location but are also determined by patient age and tumor histology [5]. Elevated circulating gonadotropin levels have been cited as a factor in their pathogenesis and in this regard GCT has been linked to certain conditions with a chromosome X overdosage (i.e., Klinefelter syndrome) [4]. Teratomas have been traditionally thought to arise by misplacement of multipotential germ cells [6].

GCT are mainly categorized according to histology, in 5 subtypes of increasingly malignant behaviour: Germinoma, Teratoma, Embryonal Carcinoma, Yolk Sac Tumor and Choriocarcinoma [2, 4]. From a therapeutic standpoint these last 3 are considered malignant [5]. Elevation in serum and CSF of tumor markers Alfa-Fetoprotein (AFP) and $\beta$-Human Chorionic Gonadotropin (HCG), can further divide intracranial GCT into 'secreting' and "no secreting" lesions [2]. Elevation of AFP $(>10 \mathrm{mcg} / \mathrm{L})$ is characteristic of Yolk Sac Tumor but may also be present in milder amounts in Teratomas with enteric components. High levels of HCG (>50 IU/L) are typically associated with Choriocarcinoma, while lower levels may be secreted by syncytiotrophoblastic elements of Germinomas [7]. NonGerminomatous Germ Cell Tumors (NGGCT) typically show significant elevations of AFP and HCG both in serum and CSF and carry a poorer prognosis. Mixed forms account for $25-32 \%$ of cases [1]. Pure germinomas account for $50 \%$ of all GCT but comprise up to $65 \%$ of pediatric intracranial GCT [5]. Teratomas, both pure and as part of mixed forms, account for $15-20 \%$ of all intracranial GCT [2, 5].

Matsunani has described a classification with three prognostic groups based on histology [8]. Pure germinoma with its remarkable radiosensitivity and pure mature teratoma that is potentially curable by gross total resection are included in the "good prognosis" group with an overall survival rate (OSR) exceeding 90\%. Immature Teratoma and Mixed forms dominated by teratoma and germinoma with limited amounts of NGGCT represent the "intermediate position" in terms of biologic potential with a OSR of approximately $70 \%$, while pure NGGCT and mixed lesions with predominance of these aggressive tumors account for the "poor prognosis" group with an OSR of less than $40 \%$ [8].

Teratomas may originate anywhere along the midline. They most commonly become implanted in the sacrococcygeal, gonadal, mediastinal, retroperitoneal, or intracranial regions. Intracranial teratomas tend to arise from midline structures with the pineal gland and suprasellar region being the most common [5]. In 5-10\% of cases, both locations can be affected simultaneously and $10 \%$ of patients have leptomeningeal or intraventricular tumor spread at the time of diagnosis [5]. In the series reviewed, 111 intracranial teratomas were reported, 11 out of them were described as originating in the third ventricular region, representing $10 \%$, compared to 48 cases affecting the pineal region or
29 to sellar and suprasellar area, which accounted for the majority of all reported intracranial teratomas. In 6 cases, teratomas in the third ventricle were described in association with teratomas in pineal or sellar region $[9,10]$. They are subclassified in mature variants containing fully differentiated "adult type" components; immature teratomas that are composed of incompletely differentiated foetal type tissues; and teratomas with malignant transformation in which an additional cancer of a somatic type is present.

Although radiological features vary with the contents, typical radiological elements have been described [2]. Teratomas tend to be nonsecreting tumors that appear as midline, solid-cystic, non-homogeneous lesions of mixed intensity signal with variable contrast enhancement and a mixture of large cysts, areas of calcification and foci of low signal attenuation, characteristic of fat $[2,11]$. Our patient showed distinctive radiological features with a well-defined, predominantly homogenous globular lesion in the roof of the third ventricle, isointense to brain parenchyma in T1WI, hyperintense in T2WI and with regular enhancement of its capsule.

However, it is impossible to make the diagnosis of a teratoma on the basis of radiology imaging alone. The definitive diagnosis of intracranial teratomas is on the basis of histopathology. Unless contraindicated, any patient with a suspected intracranial GCT should have done as part of the initial workup: cerebral and spinal MRI; measurement of AFP and HCG in both serum and CSF; cytology from CSF; and a tissue biopsy of the mass lesion [5]. Lumbar CSF has been described as more accurate for tumor markers and cytology than ventricular sources [5]. Differential diagnosis should include astrocytoma, ependymoma, primitive neuroectodermal tumor, choroid plexus papilloma, and craniopharyngioma in cases of calcified suprasellar lesions.

Neurosurgical management of GCT comprises three main issues: diversion of CFS in the setting of acute hydrocephalus; obtaining a biopsy for tissue diagnosis; and cytoreduction, having in mind that gross total resection is curative for mature teratomas, with no adjuvant therapy been necessary if complete removal has been achieved [1, 5, 12]. Radical resection is strongly advocated for intracranial teratomas because malignant transformation and recurrence after partial removal of mature teratomas has been reported [1,2]. Ventriculoperitoneal shunt has fallen out of favour as a primary option for the management of acute hydrocephalus in patients with suspected intracranial GCT, given that obstruction of CSF pathways often resolves rapidly with treatment and VPS poses a significant risk for shunt related metastases [5]. In this regard, neuroendoscopic biopsy, although reported to yield the lowest level of accuracy because of the small quantity of biopsy material sampled is also useful to restore CSF circulation and permits CSF sampling for tumor markers and cytological examination [2]. Temporary external ventricular drainage (EVD) may be useful in patients harbouring radiosensitive germinomas with a fast response to therapy, and in those for whom an open surgical resection is planned as a primary treatment.

Noudel proposes an algorithm primarily guided by tumor markers in which surgery, attempting gross total resection, should be performed as a first line therapy in non-secreting lesions with clinical and radiological evidences of teratoma [2]. From this standpoint, lesions with positive markers should undergo endoscopic or stereotactic biopsy and receive 
neoadjuvant therapy, reserving surgery for resection of residual masses after chemo-radiotherapy. Resection of these residual lesions through what has been termed second look surgery, often yields a teratomatous component or fibrosis [5]. Mature teratomas are considered to have a good prognosis, with total resection being curative. Prognosis is poor for immature teratomas and teratomas with malignant transformation, and even when totally removed, adjuvant therapy is required $[1,8,11]$.

\section{Conclusion}

Teratomas of the central nervous system are rare and are frequently found in children and young adults. They are rare lesions that account for $0.5-1 \%$ of all intracranial tumors and $2-4 \%$ of intracranial tumors in children $[6,11,13]$. Intracranial teratomas predominantly affect the pineal region and Their occurrence in the third ventricle has been described in $21 \%$ of the reported series and in most cases in pediatric population [1-3, 6, 11, 13, 14]. We describe a case of a mature teratoma arising in the third ventricle of a 37-year-old male patient. Atypical radiological features were present.

\section{REFERENCES}

1. Lee YH, Park EK, Park YS, Shim KW, Choi JU et al. (2009) Treatment and outcomes of primary intracranial teratoma. Childs Nerv Syst 25: 1581-1587. [Crossref]

2. Noudel R, Vinchon M, Dhellemmes P, Litré CF, Rousseaux P (2008) Intracranial teratomas in children: the role and timing of surgical removal. J Neurosurg Pediatr 2: 331- 338. [Crossref]

3. Kim JM, Cheong JH, Yi HJ, Bak KH, Kim CH et al. (2002) Metachronous germinoma after total removal of mature teratoma in the third ventricle: a case report. J Korean Med Sci 17: 287-291. [Crossref]
4. Rosemblum MK, Nakazato Y, Matsutani M (2007) Central Nervous System Germ Cell Tumors. The 2007 WHO Classification of Tumours of the Central Nervous System 194-204.

5. Winn HR (2011) Youmans Neurological Surgery. Sixth Edition. Elsevier Saunders.

6. Agrawal M, Uppin MS, Patibandla MR, Bhattacharjee S, Panigrahi MK et al. (2010) Teratomas in Central Nervous System: A clinicomorphological study with review of literature. Neurol India 58: 841846. [Crossref]

7. Ha BJ, Kim YS, Cheong JH (2016) Large Mature Cystic Teratoma of the Third Ventricle in Infancy: A Case Report and Review of Literatures. Brain Tumor Res Treat 4: 44-47. [Crossref]

8. Matsutani M, Sano K, Takakura K, Fujimaki T, Nakamura O et al. (1997) Primary intracranial germ cell tumors: a clinical analysis of 153 histologically verified cases. J Neurosurg 86: 446-455. [Crossref]

9. Zhao J, Wang H, Yu J, Zhong Y, Ge P (2012) Cerebral falx mature teratoma with rara imaging in an adult. Int J Med Sci 9: 269-273. [Crossref]

10. Shim KW, Kim DS, Choi JU, Kim SH (2007) Congenital Cavernous Sinus Teratoma. Yonsei Med J 48: 704-710. [Crossref]

11. Goyal N, Kakkar A, Singh PK, Sharma MC, Chandra PS et al. (2013) Intracranial teratomas in children: a clinicopathological study. Childs Nerv Syst 29: 2035-2042. [Crossref]

12. Sawamura Y, Kato T, Ikeda J, Murata JI, Tada M et al. (1998) Teratomas of the central nervous system: treatment considerations based on 34 cases. J Neurosurg 89: 728-737. [Crossref]

13. Zigourakys CC, Davis JL, Kaur G, Ames CP, Gupta N et al. (2015) Management of central nervous system teratoma. J Clin Neurosci 22: 98-104. [Crossref]

14. Zhou P, Li Y, Yang Z, Shu J (2014) Mature cystic teratoma of skull base and attached to the wall of third ventricle. Turk Neurosurg 24 292-293. [Crossref] 